\title{
Modèles de fusion de la neige en un point et leur intégration dans les modèles hydrologiques de bassin versant*
}

\author{
PAR \\ Charles Obled \\ Ingénieur à l'Institut National \\ Polytechnig̨ue de Grenoble

\section{ET Bernard Rosse} \\ Inirénieur \\ a I'Institut de Mécanique de Grenoble(1)
}

\section{Résumé}

Cet article fait le point des travaux que nous avons entrepris dans le domaine de l'hydrologie nivale, en particulier la modélisation de la fusion de la neige.

Un premier modèle, relativement sophistiqué, a été mis au point en utilisant les connaissances récentes sur les processus mis en jeu par la fonte de la neige. Ce modèle a été ajusté puis testé sur les données lysimétriques et micro-météorologiques de deux sites expérimentaux, l'un en forêt, l'autre en zone boisée, situés au col de Porte $(1350 \mathrm{~m})$ près de Grenoble. On a envisagé de façon très détaillée les hypothèses pour la modélisation, et certaines ont fait l'objet ultérieurement d'une campagne de mesures.

En utilisant un pas de temps de 3 heures, ce modèle simule de façon très satisfaisante les écoulements du lysimètre et l'état thermique du manteau de neige tout au long de la période de fonte.

Ensuite, de façon à n'utiliser que des données couramment disponibles en région de montagne, la structure du modèle a été légèrement simplifiée, les données manquantes remplacées par diverses estimations. La sensibilité du modèle à ces simplifications et estimations, et la réduction correspondante des performances est brièvement commentée.

A ce niveau de simplification, il est apparu qu'un pas de temps de 12 heures était suffisamment précis et un sous-programme, compatible avec les exigences d'un modèle complet de bassin versant a été mis au point. On en donne des exemples sur les données du col de Porte et du site expérimental de Davos (2500 m, Suisse).

Enfin, on présente quelques remarques concernant les modèles de fonte de neige les plus courants, utilisant des index de température et un "facteur de fonte". La variation de ce facteur avec les types de temps (clair, couvert ou pluvieux) a été mise en évidence et justifie quelques précautions lors de la mise en ouvre d'un tel modèle.

.......

(*) Cette communication a été publiée "in extenso" dans les cahiers de l'ORSTOM, série Hydrologie, Vol. XII, n 4 - 1976,

\section{Abstract}

Models of snow melting conditions, at a single point, and their incorporation into hydrological catchment area models.

This paper is a review of different works dealing with snow hydrology and particularly the modelling of snowmelt. The first model is a detailed one, based on present knowledge on snowmelt processes. This model was calibrated and tested on the data of two lysimeter sites, located in the North French Alps, one in an open area and one in a forested area. The assymptions required are extensively discussed and have been sustained by further measurements.

Using a 3 hours time step, this model appears to be very satisfactory to simulate meltwater outflows and the thermal status of a snowpack at the end of the accumulation period and during the whole melt period.

Then in order to fit the data usually available in mountaineous regions, the model structure was then slightly simplified, and the lacking data have been replaced by various estimations. The sensitivity of the model and the corresponding degradation of the results is shortly considered.

It appears that a subroutine with a time step of 12 hours is sufficiently accurate for the majority of watershed models require ments. Such a subroutine which can easily be integrated in a complete watershed model, is finally presented.

Finally, some remarks are presented concerning the usual snowmelt models using temperatures index and a "meltfactor". The dependence of this factor on the types of weather situation (clear or overcast sky) has been specially pointed out and some conclusions are drawn for this particular type of models.

sous le titre "Modèles mathématiques de la fusion nivale en un point". Nous n'en donnerons done qu'un résumé.

(1) Maintenant au Service Technique Central, Direction des ports et voies navigables, Compiègne. 


\section{Discussion}

Président : M.L. de CRECY

M. le Président remercie $M$. OBLED de son intéressant exposé d'hydrologie nivale. Il signale qu'à partir de sa Communication, M. OBLED a préparé un mémoire qui paraîtra dans les "Cahicrs de I'O.R.S.T.O.M.". En conséquence, ladite communication ne sera pas imprimée dans la Houille Blanche qui se bornera à publier un résumé substantiel de la discussion qui va $s$ 'instaurer.

M. REMENIERAS intervient en ces termes:

Dans sa thèse et dans un récent article $\left({ }^{1}\right), M$. de la CASINIERE a tenté de relier la fusion de la neige au bilan radiatif de la surface enneigée. Cette approche s'est-elle révélée féconde pour la mise au point de vos modeles?

Très certainement, répond $M$. OBLED, le modèle effectue un calcul complet du bilan thermique donc en partie radiatif, à l'interface air-neige. Ce calcul utilise des mesures disponibles, et à défaut, ou en complément, des lois d'estimations partiellement empiriques. Des mesures très complètes effectuées par ailleurs, comme celles de $\mathrm{M}$. de la CASINIERE, ont permis de préciser ces lois et leurs coefficients, que nous intégrons ensuite dans nos modèles. A titre d'exemple, nous avons utilisé comme valeurs d'entrée dans nos modèles, les données mesurées du rayonnement solaire. Quand nous avons essayé de supprimer dans notre modèle certaines de ces données difficiles et coûteuses à obtenir, il en est résulté une diminution inacceptable des performances. Nous avons alors tenté de remplacer les valeurs mesurées du rayonnement solaire par des estimations tirées des abaques figurant dans les annuels de météorologie ou d'hydrologie. Malheureusement, par temps plus ou moins couvert, ces estimations sont très mauvaises et entraînent des erreurs de l'ordre de $50 \%$ sur la quantité journalière de calories reçues.

M. le Président se déclare surpris par la bonne simulation sur le modèle - de la fonte des neiges en forêt ; cette dernière introduit, en effet, un facteur d'incertitude supplémentaire du fait de linterception par les arbres et de l'inégale répartition de la neige et de la fonte de celle-ci suivant les secteurs.

Peut-être, répond $M$. Ов LED, les bons résultats obtenus peuventils trouver une explication a posteriori. En forêt, les termes "turbulents" perdent de leur importance car le vent est faible dans les peuplements denses ; dans les memes conditions, le rayonnement solaire n'atteint pas le sol - même en hiver - sous une couverture de résineux. Au total, le bilan thermique sera dominé par le rayonnement infrarouge du couvert - constitué par les arbres - vers le manteau neigeux.

Or ce rayonnement est fonction de la température à la base du couvert, très voisine de la température de l'air, facile à mesurer. Le bilan thermique contient donc un terme dominant et de plus facile à estimer, d'où les bons résultats. Cela explique aussi l'étroite corrélation entre la température de l'air et les écoulements journaliers dûs à la fusion nivale dans les bassins fortement boisés. Dans les forèts comportant des arbres à feuilles caduques, le problème peut être plus complexe surtout en période hivernale, car dans la mesure où ces arbres ne couvrent pas complètement le manteau neigeux, il subsistera un fort rayonnement infra-rouge de la neige vers l'atmosphère, non compensé par celui du couvert, et le rayonnement solaire gardera un rôle important, mais difficile à estimer.

$-\cdots-\cdots-\cdots$

(1) de la CASINIERE. - Heat exchange over a melting snow surface. Journal of Glaciology, Vol. 13, 55-72, 1974.
Ne peut-on tenter, demande M. GuILlor de pallier l'absence d'enregistrement du rayonnement par une reconstitution vraisemblable de cet enregistrement à partir de l'enregistrement de la température ; ainsi, par temps clair, il y a un grand écart entre le maximum et le minimum de température ; au contraire, un ciel plus ou moins couvert réduit la valeur de l'amplitude journalière des températures.

$L a$ reconstitution du rayonnement solaire, répond $M$. OBLED, exige un processus relativement complexe et risque d'aboutir à des valeurs trop incertaines pour être utilisées dans un modèle relativement sophistiqué. Par temps clair, la valeur du rayonnement solaire, à une date déterminée, sur une surface dont la latitude, la pente et l'orientation sont connues, peut être assez bien déterminée ; il n'en est pas de même par temps couvert ou perturbé (influence de l'humidité atmosphérique, de la nébulosité, du passage d'un front, etc. .).

Vous avez surtout ajusté le modèle, remarque $M$. DELSOL, sur les données du $\mathrm{Col}$ de Porte, site relativement abrité. Avezvous fait des essais dans des zones très ventées?

D'après les essais - peu probants, il est vrai … portant sur le site de DAVOS, répond $M$. OBLED, il semble que le modèle soit transposable, en principe, à des zones venteuses. La difficulté est d'obtenir des observations de vent représentatives à l'échelle du bassin versant considéré.

\section{LEFEBVRE (SOGREAH) pose la question suivante}

"Les comparaisons présentées dans la Communication entre les valeurs calculées et mesurées font état d'un bilan cumulé des quantités d'eau écoulées en fonction des différents facteurs. . Le pas de temps et la durée totale pris en compte pour ce cumul ont-ils une influence sur les résultats et la recherche d'une optimisation de ces durées a-t-elle été tentée?

\section{OBLED répond:}

Nous initialisons le modèle un certain jour, par exemple le $1^{\text {er }}$ Mars puisque nous sommes surtout intéressés par la période de fusion de la neige (mais cette dernière peut être interrompue par de longues périodes de froid). Suivant le processus indiqué dans notre Communication, nous calculons, jour par jour, l'évolution du manteau neigeux à partir des données météorologiques et cela sur des périodes qui couvrent de 30 à 100 jours; il ne semble pas que les résultats soient moins bons au début qu'à la fin de telles périodes.

Le modèle décrit est-il bien adapté aux bassins tels que ceux du Nord Canadien dans lesquels la fusion des neiges commande le régime hydrologique des cours d'eau ? demande M. REMENIERAS

Au Canada, estime M. OBLED, les zones boisées sont extrêmement importantes et les variations d'altitude faibles, il n'est pas nécessaire d'utiliser des modèles sophistiqués - spécialement adaptés aux zones tempérées - tels que celui que j'ai présenté ; des modèles très simples basés sur le nombre de "degrés-jours" ou sur un schéma statistique élémentaire donnent de bons résultats. Il en est de même chaque fois que la fusion de la neige se fait dans des conditions homogènes :

- terrain découvert mais temps clair prolongé (Californie, Pamir) - terrain boisé en résineux

et en pratique en un seul épisode après maturation du manteau. 